\title{
РАЗРАБОТКА ИНТЕРФЕЙСА ОПЕРАТОРА КОМПЬЮТЕРНОЙ СИСТЕМЫ АВТОМАТИЗАЦИИ УСТАНОВКИ ИОННО-ПЛАЗМЕННОГО НАПЫЛЕНИЯ
}

\author{
Киркопуло Е.Г. \\ Одесский национальный политехнический университет, Одесса \\ ORCID: 0000-0001-5570-5989 \\ E-mail: kateryna.grygoryvna@gmail.com
}

Copyright $($ C) 2018 by author and the journal “Automation technologies and business - processes. This work is licensed under the Creative Commons Attribution International License (CC BY). http://creativecommons.org/licanses/by/4.0

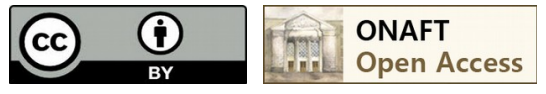

\section{DOI: $10.15673 /$ atbp.v10i4.1234}

Аннотация:Показана актуальность проблемы повышения качества работы ионно-плазменных установок. Одной из важных задач для таких установок является разработка эффективного компьютерного интерфейса их систем автоматизации. Приведены недостатки имеющихся интерфейсов. Указано, что имеющиеся интерфейсы ориентированы на логическое управление и не обеспечивают высокий уровень автоматизации процесса. Анализ технологического регламента работы установки «Булат» с разработанной для неё системой автоматического управления показал, что процесс обработки металлорежущего инструмента можно разделить на стадии со строго заданными требованиями к каждой из них. В результате анализа регламента и практики разработки эффективных компьютерных интерфейсов сформулированы требования к интерфейсам операторов ионно-плазменных установок, которые удовлетворяют требованиям ситуационной осведомленности и рекомендациям международного стандарта ANSI/ISA-101.01-2015. Приведены экраны разработанного интерфейса в различных технологических ситуациях для каждой стадии, которые иллюстрируют эффективность предлагаемого решения.

Abstract:The actuality of the problem of improving the quality of operation of ARC PVD ion-plasma installations is shown. One of the important task for such installations is the development of effective computer interface for the automation system. The drawbacks of the available interfaces are shown. It is indicated that the available interfaces are focused on logical control and do not provide a high level of process automation. The analysis of the technological regulations of the work of the "Bulat" unit with the automatic control system developed for it showed that it is possible to divide process on stages with strictly specified requirements. As a result of the analysis of the regulations and practices of developing effective computer interfaces, the requirements for interfaces of operators of ion-plasma installations are formed that meet the requirements of situational awareness and ANSI/ISA-101.01-2015 international standard recommendations. Screens of the developed interface are given in various technological situations for every stage that illustrate the effectiveness of the proposed solution.

Ключевые слова:Человеко-машинный интерфейс, компьютерная система автоматизации, ионно-плазменная установка.

Key words: Human-machine interface, computer automation system, ARC PVD ion-plasma installation.

Введение. Установки ионно-плазменного напыления предназначены для повышения износостойкости и качества работы металлорежущего инструмента Одной из наиболее совершенных технологий ионно-плазменного напыления является нанотехнология метода КИБ (катодно-ионная бомбардировка), разработанная в Украине. Такая технология используется в серийных установках - «Булат» (Украина), ННВ-6.6 (Российская Федерация), ВУ-2МБС (Беларусь), MR-333 и MR-383 (США) и другие.

C момента изобретения сама технология напыления не устарела, однако существенной модернизации требуют существующие системы автоматизированного управления технологическим процессом установки. Повышение качества работы установки ведется в трех направлениях: синтез более совершенных цифровых систем автоматического управления установкой, разработка более качественной и надежной программно-технический структуры системы автоматизации, улучшение эргономических показателей компьютерного интерфейса [1]. 
Синтез усовершенствованной поэтапной системы автоматического управления установкой проведен нами в работе [2]. Показано, что использование разработанной системы управления позволяет существенно повысить стойкость инструмента в сравнении с используемыми в настоящее время установками. Так, например, при ионной очистке удалось достичь отклонение температуры от программного значения на $5 \mathrm{~K}$ вместо регламентных $25 \mathrm{~K}$, что способствует улучшению качества обработанного инструмента, показатель дефектности которого становится равным одному баллу.

Целью настоящей работы является разработка усовершенствованного интерфейса взаимодействия с оператором. Интерфейс должен соответствовать требованиям международного стандарта ANSI/ISA-101.01-2015 и поддерживать высокий уровень автоматизации, заложенный в разработанную систему управления установкой. Разработанные ранее интерфейсы [3-10] не соответствуют предъявленным требованиям. Это объясняется тем, что задача разработки интерфейса рассматривалась отдельно от задачи разработки системы автоматического управления. Если автоматизированное управление в таких интерфейсах и реализуется, то, как правило, только с помощью алгоритмов реализации последовательного выполнения операций по заданным условиям. Из доступных источников, наиболее совершенный компьютерный интерфейс установкой ионно-плазменного напыления представлен в работе [3]. Преимуществом разработанного интерфейса является ориентация на сенсорный экран, возможность задавать рецепты, реализованная система отчетов. В то же время интерфейс реализуется не в соответствии с современными требованиями компьютерной эргономики, видеокадр экранной формы не отображает динамику процесса, а показанная система рецептов соответствует чисто логическому управлению процессом. Таким образом, задача разработки эффективного интерфейса для ионно-плазменных установок остается актуальной и в настоящее время полностью не решенной.

Основные этапы работы ионно-плазменной установки. Установка «Булат» предполагает три этапа работы: ионная очистка, нанесение покрытия и охлаждение. На каждом из указанных этапов необходима работа соответствующей системы автоматического управления. Схематически процесс может быть представлен следующим образом [1] - см. рис. 1.

Ионная очистка

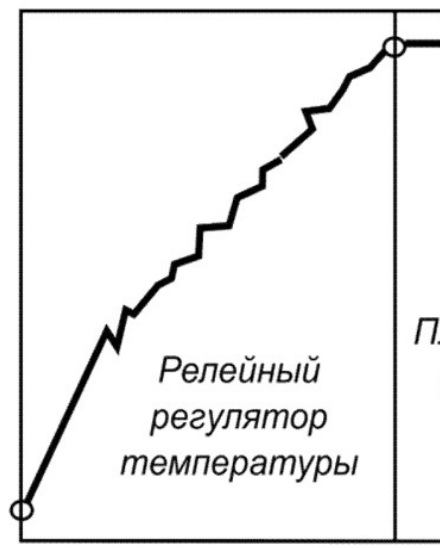

Нагрев

по программе
Нанесение покрытия

Охлаждение

Плавный регулятор температуры

Плавный регулятор давления

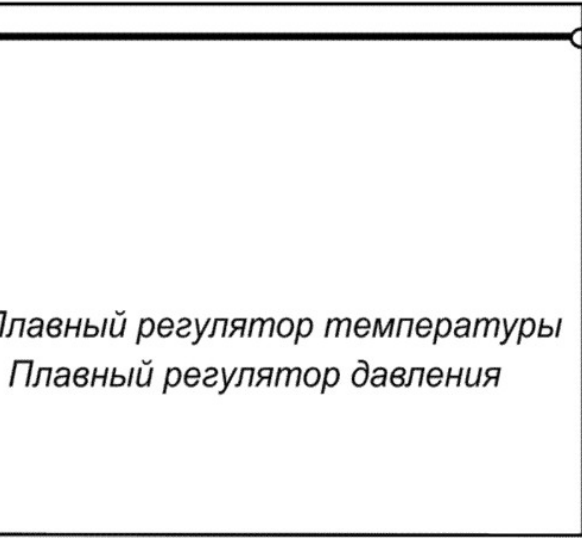

Стабилизация температуры

и давления
Плавное

охлаждение

\section{Рис. 1 - Этапы работы установки «Булат» и требуемые системы регулирования, для обеспечения качества их реализации}

Разработка систем автоматического управления для каждого из этапов проведена в работе [2]. Экспериментально определенный технологический регламент, для каждого этапа обработки стального инструмента - резца 12Х18Н10T, представлен в таблице 1.

Как видим, представленный технологический регламент позволяет характеризовать технологический процесс как строго поэтапный с жесткими временными регламентами, нарушение которого может происходить по следующим трем причинам: недостоверный регламент, некорректные настройки регуляторов, отказ оборудования. Эффективный компьютерный интерфейс должен помогать легко идентифицировать нарушение и позволять точно локализовать источник ошибки.

Формулирование требований к интерфейсу. Обзор современных стандартов в области эргономики компьютерных интерфейсов представлен в работе [11]. В работе проведена разработка интерфейса с использованием 
рекомендаций стандарта ANSI/ISA-101.01-2015 и концепции ситуационной осведомленности по модели Эндсли, который позволяет избежать распространенных ошибок проектирования ЧМИ промышленных технологических процессов. Обзор основных ошибок проектирования, которые устоялись в практике промышленной автоматизации, приведен в работе [12]. Из обзора выделим те проблемы, которые касаются распространенных компьютерных интерфейсов ионно-плазменных установок:

- на основном экране размещается мнемосхема с множеством цифровых параметров, что не позволяет оценить динамику и спрогнозировать течение процесса (поскольку тренды отображаются на другом экране, или не отображаются вовсе),

- используется множество отвлекающих факторов - избыточное цветовое оформление, 3D-тени, анимация,

- организация экранов ориентирована на представление информации разных типов и не является следствием анализа типовых действий оператора и задач, которые он должен постоянно решать,

- элементная база и цифровой алфавит интерфейсов недостаточно обоснованы, что не позволяет эффективно направлять внимание оператора.

Таблица 1 - Технологический регламент обработки резца 12Х18Н10Т

\begin{tabular}{|c|c|c|c|}
\hline Этап & Операция & Требования & Техническое обеспечение \\
\hline \multirow{5}{*}{$\begin{array}{l}\text { Ионная } \\
\text { очистка }\end{array}$} & $\begin{array}{l}\text { 1. Загрузка } \\
\text { материала }\end{array}$ & Производится вручную & $\begin{array}{c}\text { Показание установленного в камеру } \\
\text { тензодатчика не должно позволять } \\
\text { продолжить процесс без материала. }\end{array}$ \\
\hline & $\begin{array}{c}\text { 2. Достижение } \\
\text { вакуума в камере }\end{array}$ & $\begin{array}{c}\text { Достижение давления } \\
0.3 \text { Па } \\
\end{array}$ & $\begin{array}{c}\text { Включить вакуум насос до достижения } \\
\text { давления, затем отключить }\end{array}$ \\
\hline & $\begin{array}{c}\text { 3. Включение } \\
\text { механизма } \\
\text { вращения } \\
\end{array}$ & Достижение 8 об/мин & $\begin{array}{c}\text { Механизм вращения деталей, датчик скорости } \\
\text { вращения }\end{array}$ \\
\hline & $\begin{array}{c}\text { 4. Плавное } \\
\text { повышение } \\
\text { температуры }\end{array}$ & $\begin{array}{c}\text { Расчет линейной } \\
\text { программы повышения } \\
\text { температуры. } \\
\text { Повышение температуры } \\
\text { по программе за заданный } \\
\text { интервал времени в } 1800 \text { с } \\
\text { при максимально } \\
\text { допустимом отклонении в } \\
25^{\circ} \mathrm{C} \text { до достижения } \\
\text { температуры в } 527^{\circ} \mathrm{C} . \\
\end{array}$ & $\begin{array}{c}\text { Система подачи напряжения, пирометр, } \\
\text { программно реализованный релейный } \\
\text { регулятор температуры. }\end{array}$ \\
\hline & $\begin{array}{l}\text { 5. Завершение } \\
\text { этапа }\end{array}$ & $\begin{array}{c}\text { Отключение регулятора и } \\
\text { системы подачи } \\
\text { напряжения } \\
\end{array}$ & $\begin{array}{c}\text { Система подачи напряжения, программно } \\
\text { реализованный релейный регулятор }\end{array}$ \\
\hline \multirow{4}{*}{$\begin{array}{l}\text { Нанесение } \\
\text { ионно- } \\
\text { плазмен- } \\
\text { ного } \\
\text { покрытия }\end{array}$} & $\begin{array}{l}\text { 1. Включение } \\
\text { подачи азота }\end{array}$ & $\begin{array}{l}\text { Открытие вентиля подачи } \\
\text { азота на уровне } 2.3 \text { мг/сек }\end{array}$ & Исполнительный механизм подачи азота \\
\hline & 2. Включение дуги & $\begin{array}{c}\text { Включение системы } \\
\text { подачи напряжения на } \\
\text { дугу с начальным } \\
\text { напряжением } 362 \text { В. } \\
\end{array}$ & Блок регулятора напряжения дуги \\
\hline & $\begin{array}{l}\text { 3. Стабилизация } \\
\text { давления и } \\
\text { температуры в } \\
\text { камере }\end{array}$ & $\begin{array}{c}\text { Обеспечение стабильной } \\
\text { температуры и давления в } \\
\text { течение } 1000 \text { сек с } \\
\text { помощью включения } \\
\text { соответствующих ПИ- } \\
\text { регуляторов }\end{array}$ & $\begin{array}{c}\text { Манометр, исполнительный механизм подачи } \\
\text { азота, пирометр, блок регулятора напряжения } \\
\text { дуги, программно реализованные ПИ- } \\
\text { регуляторы }\end{array}$ \\
\hline & $\begin{array}{l}\text { 4. Завершение } \\
\text { этапа }\end{array}$ & $\begin{array}{c}\text { Отключение регуляторов и } \\
\text { системы подачи } \\
\text { напряжения на дугу } \\
\end{array}$ & $\begin{array}{c}\text { Блок регулятора напряжения дуги, } \\
\text { программно реализованные ПИ-регуляторы }\end{array}$ \\
\hline \multirow[t]{2}{*}{$\begin{array}{l}\text { Охлажде- } \\
\text { ние }\end{array}$} & $\begin{array}{l}\text { 1. Плавное } \\
\text { охлаждение }\end{array}$ & $\begin{array}{c}\text { Включение регулятора и } \\
\text { охлаждение до } 227^{\circ} \mathrm{C} \text { в } \\
\text { течение } 1800 \text { сек при } \\
\text { обеспечении скорости } \\
\text { изменения температуры не } \\
\text { более } 0.3^{\circ} \mathrm{C} / \mathrm{ce \kappa} \\
\end{array}$ & $\begin{array}{c}\text { Пирометр, механизм подачи азота, } \\
\text { программно реализованный ПИ-регулятор }\end{array}$ \\
\hline & 2. Отключение & Отключение регулятора и & Программно реализованный ПИ-регулятор, \\
\hline
\end{tabular}




\begin{tabular}{|c|c|c|c|}
\hline & $\begin{array}{c}\text { регулятора } \\
\text { охлаждения и } \\
\text { системы подачи } \\
\text { азота }\end{array}$ & $\begin{array}{c}\text { закрытие вентиля подачи } \\
\text { азота }\end{array}$ & механизм подачи азота \\
\cline { 2 - 4 } & $\begin{array}{c}\text { 3. Завершение } \\
\text { этапа }\end{array}$ & $\begin{array}{c}\text { Достижение атмосферного } \\
\text { давления в камере и } \\
\text { отключение установки. }\end{array}$ & Манометр, выключатель напряжения. \\
\hline
\end{tabular}

Сведем рациональные требования и способы их реализации в таблицу 2.

Таблица 2 - Основные требования к разработке интерфейса и способы их реализации

\begin{tabular}{|c|c|}
\hline Требование & Способ реализации \\
\hline $\begin{array}{c}\text { Использование визуального } \\
\text { представления экранов в } \\
\text { соответствии с } \\
\text { рекомендациями стандарта } \\
\text { ANSI/ISA } 5.1\end{array}$ & $\begin{array}{ll}\text { 1. } & \text { Используется светло-серый фон } \\
\text { 2. } & \text { Используется упрощенная мнемосхема установки с использованием } \\
\text { темно-серых линий } \\
\text { 3. } \\
\text { Желтый цвет закреплен за предаварийной, красный - за аварийной } \\
\text { сигнализацией } \\
\text { 4. Действия операторов должны подтверждаться }\end{array}$ \\
\hline $\begin{array}{c}\text { Достижение ситуационной } \\
\text { осведомленности }\end{array}$ & $\begin{array}{ll}\text { 1. } & \text { Основные параметры отображаются в трендах с указанием } \\
\text { допустимых границ параметров пунктирными линиями темно- } \\
\text { зеленого цвета, что позволяет спрогнозировать течение процесса } \\
\text { 2. } \\
\text { Тренд отображается не за весь промежуток процесса, а за } \\
\text { рациональный, который позволяет оценить ситуацию - в данном } \\
\text { случае } 10 \text { мин } \\
\text { 3. } \\
\text { Состояние выполнения текущего этапа процесса можно оценить с } \\
\text { помощью полоскового индикатора }\end{array}$ \\
\hline $\begin{array}{c}\text { Логически обоснованная } \\
\text { организация экранов с } \\
\text { ориентацией на задачи, } \\
\text { которые должны решаться } \\
\text { оператором }\end{array}$ & $\begin{array}{ll}\text { 1. } & \text { Выделяется } 5 \text { экранов: процесс, отчеты, рецепт, диагностика, } \\
\text { настройка } \\
\text { 2. } \\
\text { Все экраны имеют три обязательные зоны: меню переключения } \\
\text { экранов, основная зона, зона отображения сообщений } \\
\text { 3. } \text { Экран «процесс» предназначен для просмотра состояния течения } \\
\text { процесса, его содержимое динамически изменяется в зависимости от } \\
\text { текущего этапа технологического процесса. }\end{array}$ \\
\hline $\begin{array}{c}\text { Использование интуитивно } \\
\text { понятной элементной базы } \\
\text { интерфейса, которая } \\
\text { соответствует цветовому } \\
\text { алфавиту }\end{array}$ & $\begin{array}{l}\text { 1. Цвет линий упрощенной мнемосхемы и цвет текста её элементов - } \\
\text { темно-серый } \\
\text { 2. Для отображения связи элементов контуров управления используется } \\
\text { пунктирная линия черного цвета. } \\
\text { 3. Для отображения активных элементов интерфейса (датчиков, } \\
\text { исполнительных механизмов, ползунка прогресса) используется } \\
\text { синяя линия с закраской белым цветом. } \\
\text { 4. } \text { Цвет текста активных технологических параметров - синий. } \\
\text { 5. } \text { Цвет текста параметров, которые подлежат вводу - светло-зеленый } \\
\text { 6. } \text { Цвет текста элементов, по которым можно щелкнуть, - фиолетовый. }\end{array}$ \\
\hline
\end{tabular}

Разработка интерфейсных экранов. Рассмотрим реализацию каждого из пяти интерфейсных экранов. Как указано в табл. 2, разрабатываемый интерфейс должен состоять из 5 экранов.

Экран «Рецепт» позволяет создать или редактировать текущий рецепт. Рецепт задается для заданного типа материала и партии. Целью формирования рецепта является достижение заданных показателей качества продукции, в первую очередь - стойкости, микротвердости и толщины покрытия инструмента. Для определения этих параметров до работы инструмента возможно использовать формулы, приведенные в работе [13]. Рассмотрим определение параметров инструментов взятого нами материала - металлического резца 12Х18Н10Т. Стойкость инструмента зависит от параметров технологического процесса - давления Р, Па, температуры T, K, и времени нахождения т, с в ионизационной камере. Тогда, стойкость инструмента определяется по формуле

$C=0.12 \cdot \sigma \cdot 10^{-6}+1.31 \cdot H+4.24 \cdot \delta=0.12 \cdot 4.30 \cdot 10^{-1}+1.31 \cdot 31.72+4.24 \cdot 6.78=74.24$ мин, где сцепление с покрытием $\sigma$ равно

$$
\begin{aligned}
& \quad \sigma=4.3 \cdot 10^{5}-\left(10^{5} \cdot\left(3.1 \cdot 10^{-3} \cdot(T-786)\right)^{2}\right) \cdot\left(2-e^{0.19 \cdot p}\right)=4.3 \cdot 10^{5}-\left(10^{5} \cdot\left(3.1 \cdot 10^{-3} \cdot(T-786)\right)^{2}\right) \cdot\left(2-e^{0.190 .3}\right)= \\
& \quad=4.3 \cdot 10^{5} \text { Па }=0.43 \text { МПа } \\
& \text { микротвердость инструмента равна }
\end{aligned}
$$




$$
\begin{aligned}
& H=32-\left(1 \cdot 10^{-4} \cdot(800-728 \cdot(1+0.08 \cdot P))^{2}\right) \cdot\left(2-e^{0.22 \cdot P}\right)=32-\left(1 \cdot 10^{-4} \cdot(800-728 \cdot(1+0.08 \cdot 0.3))^{2}\right) \cdot\left(2-e^{0.220 .3}\right)= \\
& =31.72 \text { ГПа, } \\
& \text { толщина покрытия инструмента равна } \\
& \quad \delta=1.111 \cdot 10^{-9} \cdot \tau \cdot\left(7.2-e^{0.32 \cdot P}\right)=1.111 \cdot 10^{-9} \cdot 1000 \cdot\left(7.2-e^{0.32} 0.3\right)=6.78 \cdot 10^{-6} \mathrm{M} .
\end{aligned}
$$

Следовательно, уже при формировании рецепта можно предварительно оценить параметры качества. По результатам функционирования установки достигнутые параметры качества можно оценить, используя средние значения технологических параметров (значения параметров качества показаны на форме этапа охлаждения). Внешний вид экрана «Рецепт» показан на рис. 2.

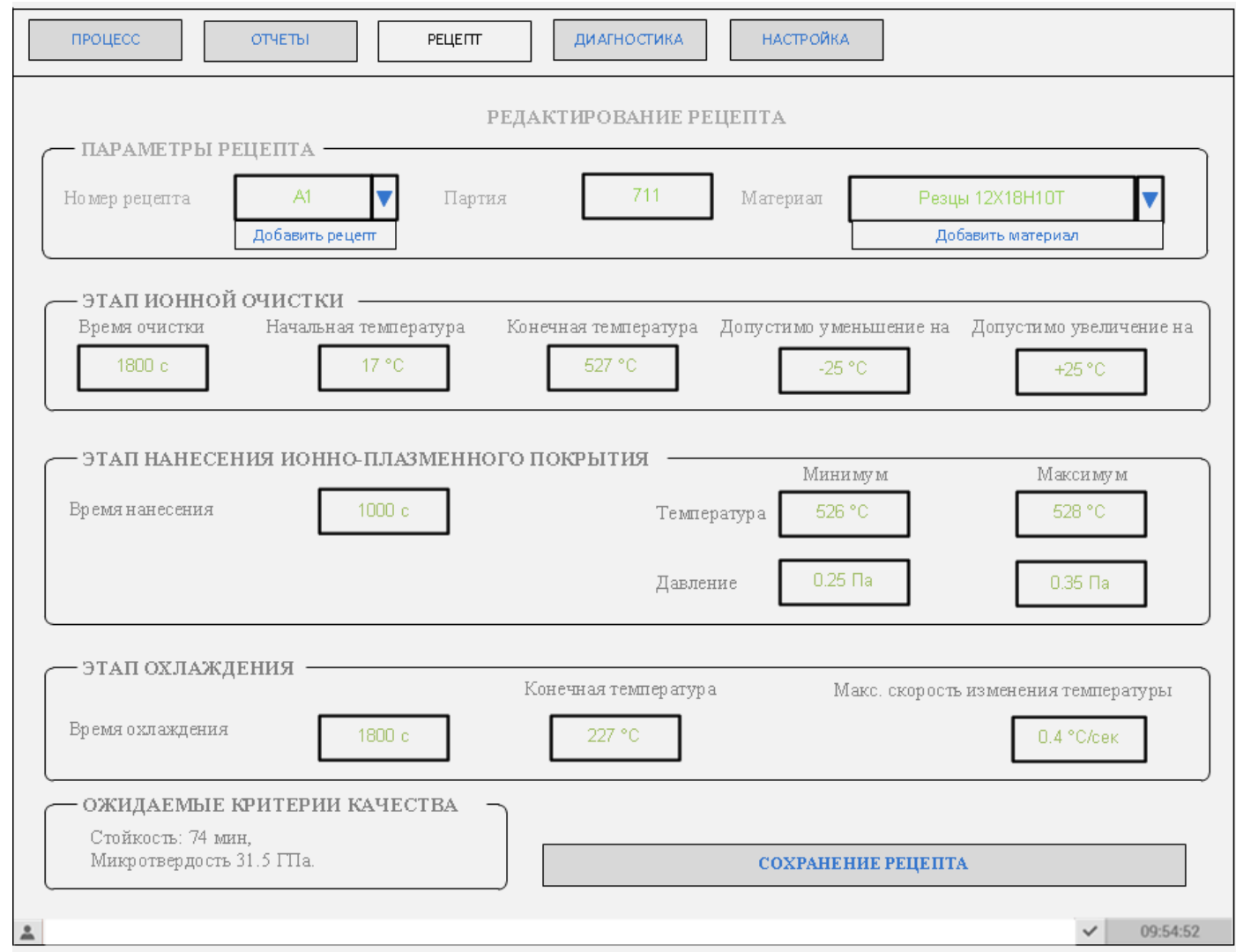

Рис. 2 - Внешний вид экрана «Рецепт»

Экран «Процесс» используется для наблюдения за ходом выполнения всех этапов технологического процесса, который выполняется в соответствии с заданным в рецепте регламентом. Внешний вид экрана на первом этапе показан на рис. 3.

На рис. 3 показана технологическая ситуация превышения температуры при выполнении операции плавного повышения температуры на 1 этапе (ионной очистки). Управляемая переменная регулятора температуры (РТ1) достигла $207^{\circ} \mathrm{C}$, что превышает требуемое на 600 с управляющей программой значение в $177 \pm 25^{\circ} \mathrm{C}$. Сообщение об этом отображается снизу экрана вместе с подачей звуковой сигнализации, которая продолжается до подтверждения сообщения. На рис.4 показан внешний вид указанного экрана на 2 и 3 этапе. На рис. 4, а показана технологическая ситуация отказа двигателя исполнительного механизма подачи азота в камеру при выполнении операции стабилизации давления и температуры в камере на 400 с. На рис 4, б показано нормальное выполнение операции плавного охлаждения 3 этапа технологического процесса на 1600 с. Поскольку этап нанесения покрытия завершен, то на экране возможно вывести рассчитанные показатели исходя из фактических отклонений параметры температуры и давления. 


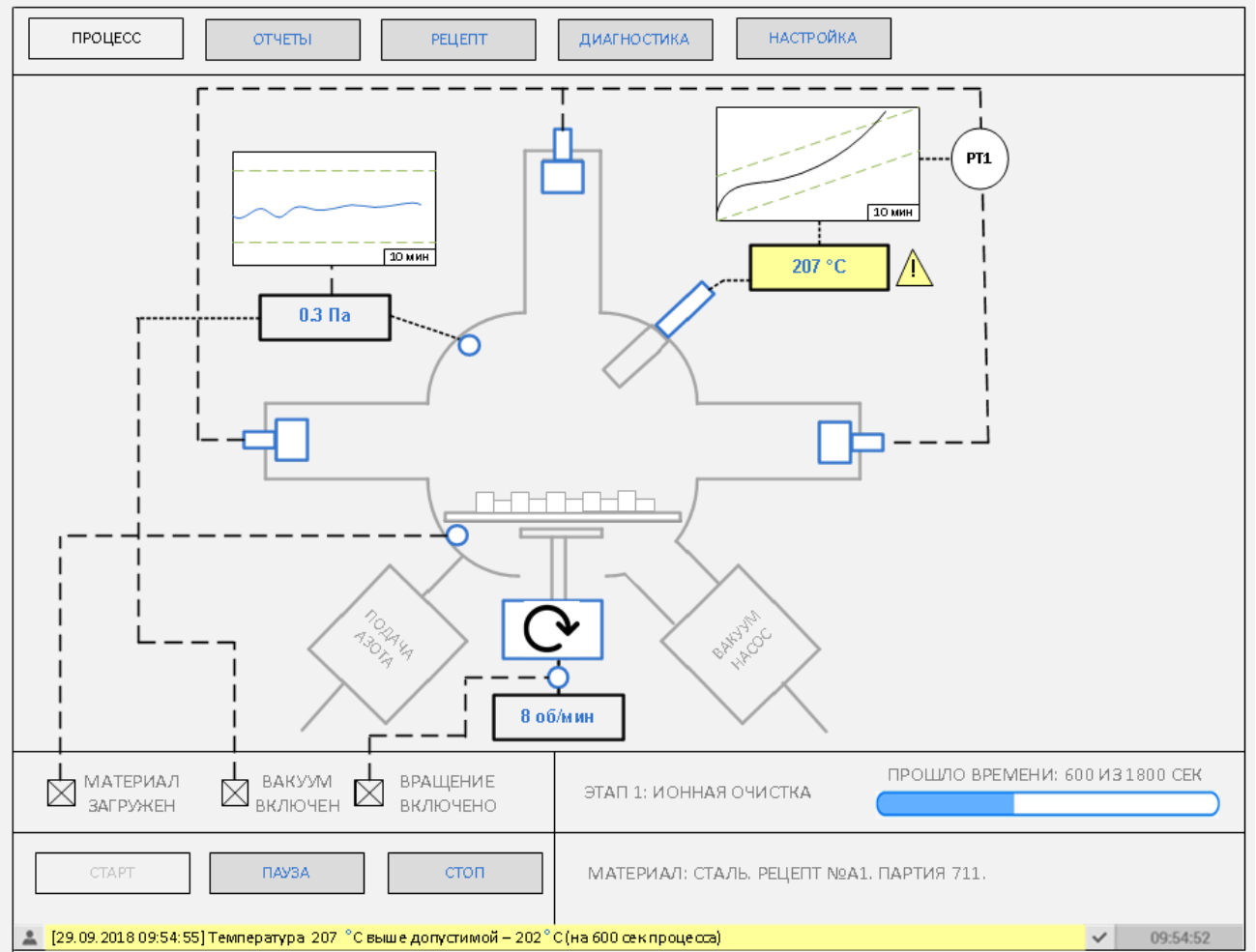

Рис. 3 - Внешний вид экрана «Процесс» на 1 этапе

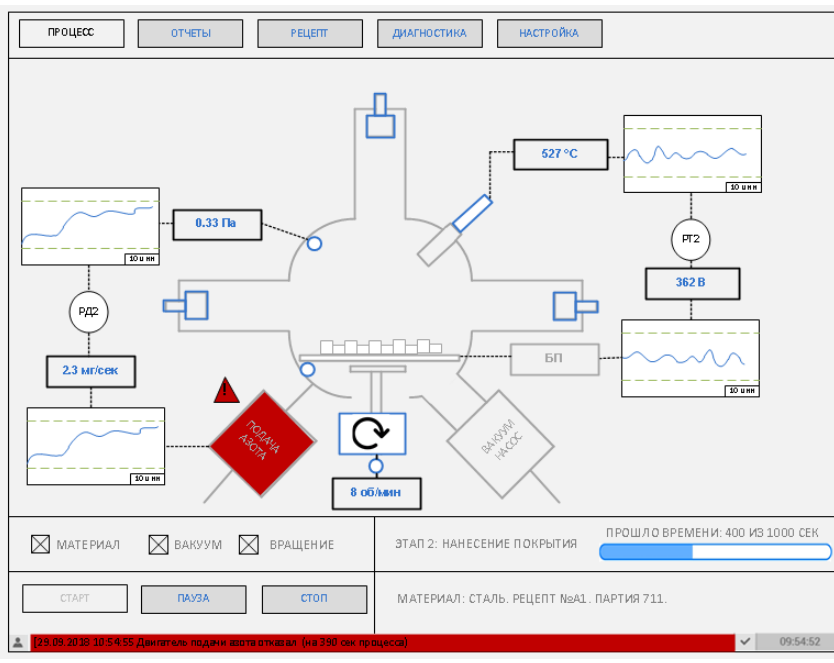

а) Этап нанесения покрытия

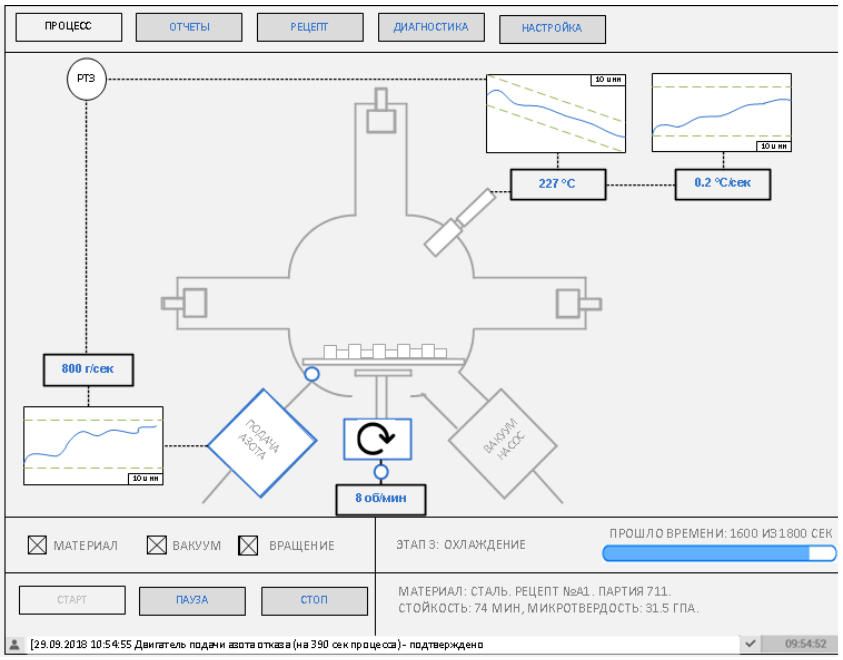

б) Этап охлаждения

Рис. 4 - Внешний вид экрана «Процесс» на 2 и 3 этапе

Экран «Диагностика» предназначен для выполнения диагностики функций системы автоматизации и для вмешательства в ход технологического процесса. При возникновении аварийной или передаварийной технологической ситуации оператор имеет три варианта действий: останов процесса, игнорирование сообщения до конца работы процесса (в случае предупреждений или отказа некоторого оборудования в конце этапа процесса), пауза процесса. В случае выбора паузы с помощью экрана диагностики возможно скорректировать управляющие воздействия и продолжить выполнение процесса. Внешний вид экрана показан на рис. 5,а.

Экран «Настройка» предназначен преимущественно для инженера по автоматизации и позволяет изменить настройки регуляторов. Внешний вид экрана показан на рис. 5, б. 


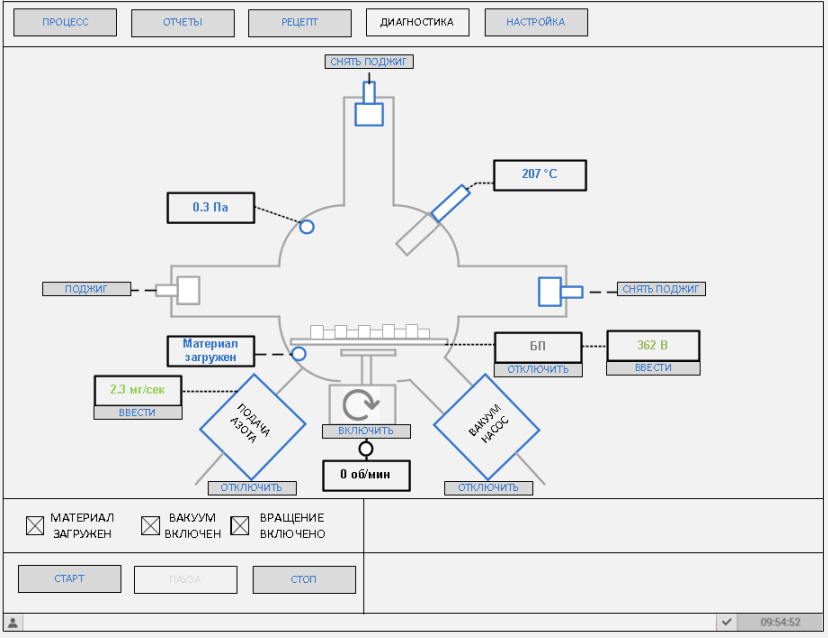

а) Экран «Диагностика»

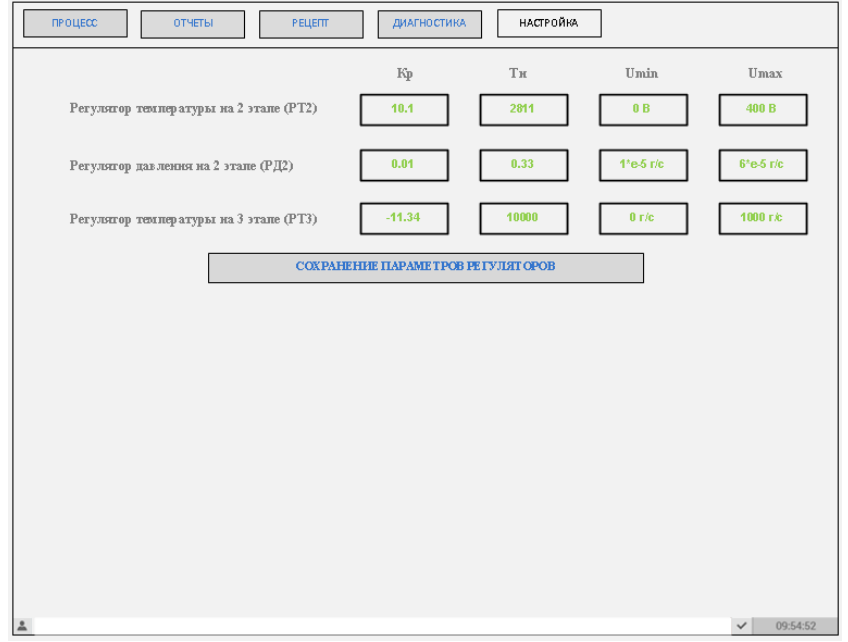

б) Экран «Настройка»

Рис. 5 - Внешний вид экранов «Диагностика» и «Настройка»

Экран «Отчеты» должен позволять отображать отчеты по изготовлению каждой партии, которые включают временные графики изменения параметров в каждом этапе, действия алгоритмов системы управления, действия операторов, возникшие ошибки, ожидаемые и достигнутые показатели качества продукции.

\section{Выводы.}

Проанализировано текущее состояние проблемы разработки эффективных интерфейсов для управления ионноплазменными установками. Показано, что имеющиеся интерфейсы не соответствуют современным требованиям эргономики и предполагают чисто логическое управление процессом. На основе анализа работы установки «Булат» приведен технологический регламент процесса обработки металлорежущего инструмента, который является строго поэтапным с жесткими временными регламентами. Оптимальным решением является использование систем автоматического регулирования для каждого из этапов процесса, синтез которых проведен в работе [2]. Анализ регламента и современных практик разработки эффективных интерфейсов позволили сформировать требования к интерфейсу оператора ионно-плазменной установки, которая функционирует с использованием разработанных систем управления.

Разработаны экраны, которые соответствуют требованиям к интерфейсу. Приведен внешний вид экранов «Процесс», «Рецепт», «Диагностика» и «Настройка» в разных технологических ситуациях. Сформированы требования к экрану «Отчет». Дальнейшим направлением исследования является вопрос программно-технической реализации разработанных систем управления и интерфейса с помощью SCADA-системы.

\section{Список использованных источников}

[1]. Киркопуло К.Г. Разработка системы автоматизации ионно-плазменной установки// Вимірювальна та обчислювальна техніка в технологічних процесах: Матеріали XVIII міжнар. наук.-техн. конференції, 8-13 червня 2018 р. Одеса. 2018. С.110-113.

[2]. Kyrkopulo K., Tonkonogyi V., Stopakevych O., Stopakevych A. Design of a set of nonlinear control systems of the arc PVD ion plasma installation//Eastern-European Journal of Enterprise Technologies. 2018. № 2 (92). C. 65-74.

[3]. Бодягин А. Автоматизированная система управления работой установки ионно-плазменного напыления в вакууме МАП-2// Современные технологии автоматизации. 2009. №3. C.52-56.

[4]. García J.A., Martínez R., Rico M., Rodríguez R. Decorative electric arc PVD coatings// Proceedings of 48th Annual Technical Conference of Society of Vacuum Coaters, April 23 - 28 2005. Denver. 2005. P. 588-591.

[5]. CatArc 2500 PVD Coating System. Brochure// Vergason Technology INC. 2018. 8p.

[6]. Mazurkiewicz A., Smolik J. Development of Novel Nano-Structure Functional Coatings with the Use of the Original Hybrid Device// Materials Science Forum. 2011. V.674. P.1-9

[7]. Yan Wenjun, Xu Qiao. Application of temperature control system based on precision control of PLC and touch screen// Issues on Modern Machines Mechanics:Proceedings of the VII International Scientific Conference, 25- 30 June 2018. Ulan-Ude. 2018. V.2. P.25-28.

[8]. The 2016 International Manufacturing Technology Show (IMTS) at McCormick Place in Chicago. Brochure. 2016. $15 p$. 
[9]. Cczk Vacuum Physical Vapor Deposition (PVD) Thin Film Coating System, Equipment// Wenzhou Cicel Vacuum Machine Co., China. Brochure. 2018. 6p.

[10]. Автоматизированной система управления вакуумной установкой ионно-плазменного азотирования ИМПЛАЗ 1000// НПЦ «Скада». Брошюра. 2018. 8с.

[11]. Арабаджи А.Ф., Стопакевич А.А., Стопакевич А.А. Разработка современного человеко-машинного интерфейса в АСУТП на основе международных стандартов // Автоматизація технологічних і бізнес-процесів. 2017. T.10. №4. С.37-46.

[12]. Стопакевич А.О. Аналіз недоліків типової практики розробки комп’ютерних людино-машинних інтерфейсів промислових систем автоматизації// 72 науково-технічна конференція ОНАЗ ім. О.С. Попова, 13-15 вересня 2015. Одеса, 2017. С.45-47.

[13]. Тонконогий В.М., Оборский Г.А. Работоспособность и надежность инструментов с износостойкими покрытиями // Труды Одесского политехнического университета. 1997. № 1 (7). С. 130-133.

\section{References}

[1]. K. Kyrkopulo, "Razrabotka systemy avtomatyzacyy yonno-plazmennoj ustanovky", in "Vymirjuvaljna ta obchysljuvaljna tekhnika $v$ tekhnologhichnykh procesakh": Materialy XVIII mizhnar. nauk.-tekhn. konferenciji, Odessa, 2018, pp. 110-113.

[2]. K. Kyrkopulo, V. Tonkonogyi, O. Stopakevych and A. Stopakevych, "Design of a set of nonlinear control systems of the arc PVD ion plasma installation", Eastern-European Journal of Enterprise Technologies, vol. 2, no. 292, pp. 65-74, 2018.

[3]. A Bodjaghyn. “Avtomatyzyrovannaja systema upravlenyja rabotoj ustanovky yonno-plazmennogho napыlenyja v vakuume MAP-2”. Sovremennye tekhnologhyy avtomatyzacyy, no. 3, pp.52-56, 2009.

[4]. J.A. García, R. Martínez, M. Rico \& R. Rodríguez. "Decorative electric arc PVD coatings", in Proceedings of 48th Annual Technical Conference of Society of Vacuum Coaters, Denver, 2005, pp. 588-591.

[5]. CatArc 2500 PVD Coating System. Brochure. Vergason Technology INC, 2018, 8 p.

[6]. A. Mazurkiewicz, J. Smolik. "Development of Novel Nano-Structure Functional Coatings with the Use of the Original Hybrid Device”, Materials Science Forum, vol. 674, 2011, pp.1-9

[7]. Yan Wenjun, Xu Qiao. "Application of temperature control system based on precision control of PLC and touch screen” in "Issues on Modern Machines Mechanics:Proceedings of the VII International Scientific Conference”, v. 2, Ulan-Ude, 2018, pp.25-28.

[8]. The 2016 International Manufacturing Technology Show (IMTS) at McCormick Place in Chicago. Brochure, 2016, $15 p$.

[9]. Cczk Vacuum Physical Vapor Deposition (PVD) Thin Film Coating System, Equipment. Wenzhou Cicel Vacuum Machine Co., China. 2018, 6 p.

[10]. Avtomatyzyrovannoj systema upravlenyja vakuumnoj ustanovkoj yonno-plazmennogho azotyrovanyja YMPLAZ 1000. NPC «Skada». 2018, 8 p.

[11]. A.F. Arabadzhy, A.A. Stopakevych, A.A. Stopakevych. "Razrabotka sovremennogho cheloveko-mashynnogho ynterfejsa v ASUTP na osnove mezhdunarodnыkh standartov”, Avtomatyzacija tekhnologhichnykh i biznes-procesiv. No. 4(10), pp. 37-46, 2017.

[12]. A.O. Stopakevych. "Analiz nedolikiv typovoji praktyky rozrobky komp'juternykh ljudyno-mashynnykh interfejsiv promyslovykh system avtomatyzaciji” in 72 naukovo-tekhnichna konferencija ONAZ im. O.S. Popova, 2015, pp.45-47.

[13]. V.M. Tonkonoghyj, Gh.A. Oborskyj. "Rabotosposobnostj y nadezhnostj ynstrumentov s yznosostojkymy pokrytyjamy, Trudy Odesskogho polytekhnycheskogho unyversyteta. No 1(7), pp. 130-133, 1997. 